\title{
Comparison of the Feasibility and Safety of Nasotracheal Suctioning With Curved Edge Catheter Versus Conventional Suction Catheter in Critically Ill Subjects: A Prospective Randomized Crossover Trial
}

\author{
Konstantinos E Grigoriadis PT, Dimitrios C Angouras MD, Aikaterini Flevari MD, and \\ Theodoros Xathos MD
}

\begin{abstract}
BACKGROUND: Nasotracheal suctioning (NTS) is accomplished by inserting a suction catheter into the trachea through the nasopharynx. It is a useful procedure in critically ill patients whose ability to cough and mobilize secretions is impaired. It was assumed that using a suction catheter with an angular tip would facilitate entry into the trachea. The primary outcome was the success rate and the ease of insertion by using a curved edge catheter (Tiemann type) compared with a conventional suction catheter. The secondary outcome was the monitoring of subject's vital signs during the intervention. METHODS: Non-intubated subjects hospitalized in 2 adult ICUs underwent 2 consecutive NTSs each, using either a 14 French curved edge catheter or a 14 French conventional suction catheter, randomly. RESULTS: Twenty subjects with a mean age of $75.5 \mathrm{y}$ were enrolled for a time period of 5 months. The tracheal access success rate was 19/52 (successful/unsuccessful attempts) using a curved edge catheter $(36.5 \%, 95 \%$ CI $23.6-51.0 \%)$ compared with $12 / 130(9.2 \%, 95 \%$ CI $4.8-15.5 \%)$ using a conventional suction catheter. The insertion was 5.6 times more likely to be achieved by using a curved edge catheter (odds ratio $5.66,95 \%$ CI 2.49-12.84, $P<.001)$. The number of attempts required to succeed in the insertion was significantly lower when using a curved edge catheter than when using a conventional suction catheter (for nasopharynx, median [range] of 1 [1] versus 2.5 [8], $P=.001$; for trachea, median [range] of 2 [9] versus $9[9], P=.002)$. The time required for successful insertion into the nasopharynx and trachea was significantly shorter when using a curved edge catheter than when using a conventional suction catheter (for nasopharynx, median [range] of $3[11] \mathrm{s}$ versus $5.3[18] \mathrm{s}, P=.038$; for trachea, median [range] of 6 [27] s versus 20 [25] s, $P=.002$ ). The traumatic rate (percentage of catheters with blood present on the tip) was exactly the same for both catheters (30\%). CONCLUSIONS: It is more likely that tracheal access will be achieved using a curved edge catheter. A shorter process time and fewer attempts are required for successful NTS using a curved edge catheter, and it seems to be an equally safe procedure. (ClinicalTrials.gov registration NCT02261428.) Key words: nasotracheal suctioning; Tiemann catheter; suction catheter; tracheal access. [Respir Care 2015;60(12):1826-1833. ( 2015 Daedalus Enterprises]
\end{abstract}

\section{Introduction}

The mucociliary transport mechanism is the normal process by which bronchial secretions are mobilized. When

Mr Grigoriadis is affiliated with the Department of Physical Therapy, Attikon University Hospital, Athens, Greece. Dr Angouras is affiliated with the Department of Cardiac Surgery, and Dr Flevari is affiliated with the Second Department of Critical Care Medicine, University of Athens, Faculty of Medicine, Attikon University Hospital, Athens, Greece. Dr this transport mechanism is impaired, the cough reflex remains the only way by which secretions can be mobilized. In

\footnotetext{
Xathos is affiliated with the University of Athens, Faculty of Medicine, Athens, Greece.

The authors have disclosed no conflicts of interest.

Correspondence: Konstantinos Grigoriadis PT, 1 Rmini str., 12462 Athens, Greece. E-mail: grigoriakost@gmail.com.
}

DOI: $10.4187 /$ respcare.03875 


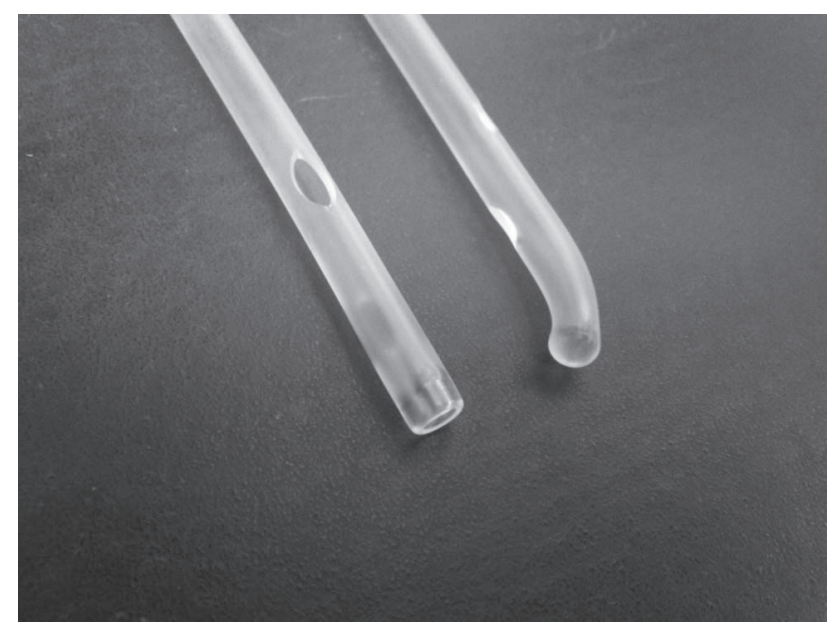

Fig. 1. Conventional suction catheter (CSC) (left) and curved edge catheter (CEC) (right).

clinical practice, especially in ICU settings, patients often have excess airway secretions, depressed cough reflex, or both. ${ }^{1,2}$ In this case, the respiratory therapist uses various therapeutic approaches to mobilize secretions. These include postural, vibrating, and breathing techniques as well as tracheobronchial tree suctioning, $, 3,4$ Tracheobronchial suctioning is an invasive procedure by which excess secretions or gastric aspirates can be removed from the trachea, especially when their presence causes physical distress or physiological impairment.

Nasotracheal suctioning (NTS) is a special technique that uses the nasal cavity as a route for the insertion of a suction catheter into the trachea through the larynx. Suction catheters commonly used in NTS have a straight and round distal tip, which ensures atraumatic introduction. Nasotracheal catheter insertion is a blind technique, closely resembling a difficult airway management technique in which a gum elastic bougie with a coudé angled tip is inserted in the trachea in a more or less blind way. ${ }^{5-7}$

Considering the above, we hypothesized that using a nasotracheal suction catheter, with an angled instead of a straight tip, would facilitate tracheal access in a carefully selected ICU patient population with difficulty in managing bronchial secretions. A curved edge catheter (Tiemann type) is a single-use catheter with an angular tip, frequently used by urologists to facilitate catheterization of a male U-shaped bulbar urethra. The term Tiemann catheter (George Tiemann \& Co., Hauppauge, NY) is being used universally to describe urological catheters with an angular tip (Fig. 1). ${ }^{8}$

Data from past literature are lacking, apart from a brief report by Pode and Manny ${ }^{9}$, which claimed that a curved edge catheter was generally more effective than a standard catheter in accomplishing tracheal access in 120 postoperative subjects. As far as we know, this is the first study

\section{QUICK LOOK}

\section{Current knowledge}

Nasotracheal suctioning (NTS) is a special technique that uses the nasal cavity as a route for the insertion of a suction catheter into the trachea through the larynx. Suction catheters commonly used in NTS have a straight and round distal tip that ensures atraumatic introduction. Nasotracheal catheter insertion is a blind technique and requires caregiver experience and skill to perform reliably.

\section{What this paper contributes to our knowledge}

In a small group of recently extubated subjects, tracheal access rates were greater using a curved edge catheter rather than a standard suction catheter. A shorter process time and fewer attempts were required for successful NTS using the curved edge catheter. There were no differences in complications between catheters, implying similar safety profiles.

comparing a curved edge catheter with a conventional suction catheter as a means for more successful NTS.

The aim of our study was to evaluate the feasibility of tracheal access (success rate of tracheal insertion and the time and number of attempts needed) by using a curved edge catheter, compared with a conventional suction catheter. The question posed in the study is clinically relevant for finding alternative and effective ways to suction.

\section{Methods}

\section{Design, Setting, and Ethical Standards}

This was a crossover clinical trial in which all subjects underwent sequential NTS by using 2 different catheters in a random order. ${ }^{10}$ The study was conducted in the Cardiothoracic and General ICU Departments of General University Hospital ATTIKON from May 2014 through September 2014 (5 months), in accordance with the principals enunciated in the World Medical Association Declaration of Helsinki. ${ }^{11}$ All manipulations were part of routine treatment, implemented under emergent conditions, and involved no more than minimal risk for the subjects, whose anonymity was preserved during data collection. The study was approved by the hospital's ethics committee (approval 9-13-16/05/14), which for the aforementioned reasons waived the need for obtaining informed consent.

\section{Subjects}

All subjects included in the study were breathing spontaneously and had respiratory failure (hypoxemia, 
hypercapnia, or both) and impaired cough reflex caused by various pathophysiological mechanisms, among which the most common was the critical illness polyneuropathy.

Because catheterization is an invasive procedure, other methods were first tried to remove secretions, depending on the condition of the subject, such as: (1) cough encouragement; (2) active cycle breathing techniques (directed cough technique or relaxed diaphragmatic breathing and deep breathing cycles followed by forced exhalation technique); and (3) chest physiotherapy (external chest wall manipulation, which includes percussion, vibration, and/or postural drainage therapy). It is noted that after any of the above physical methods and before any suctioning there was an appropriate washout period to ensure sufficient time for the subject's vital signs to return to their initial value. The washout period between suctioning was approximately $5 \mathrm{~min},{ }^{12,13}$ Taking this into account, it was assumed that $30 \mathrm{~min}$ would be more than enough time as a rest period for the participants as well as sufficient time for subject's vital signs to return to their initial value.

Inclusion criteria were: (1) spontaneous breathing without an artificial airway but with a nasogastric tube as a part of routine equipment; (2) difficulty or inability to mobilize and expectorate secretions; (3) failure of all other methods to remove secretions, and (4) presence of secretions in the trachea causing physical distress and physiological impairment (eg, impaired arterial blood gases, reduced $\mathrm{S}_{\mathrm{pO}_{2}}$, and/or increased breathing frequency).

Subjects were excluded if they: (1) were actively bleeding from the nose, (2) had severe coagulopathy (international normalized ratio value $>3.0$, platelets $<50,000)^{14}$; (3) had laryngospasm or bronchospasm; (4) had recent tracheal and/or esophageal surgery; (5) were hemodynamically unstable; (6) had a history of recent head, facial, or neck trauma; or (7) were under any sedation.

\section{Materials}

An all-purpose polyvinyl chloride conventional suction catheter 14 French (Jimit Medico Surgicals, Ahmedabad, India) and the same size (14 French) polyvinyl chloride curved edge catheter (Jimit Medico Surgicals) were used. It was necessary to have a standard size of catheter that could be applicable to all of the catheterizations, 14 French caliber was selected because it is an average size commonly used in the ICU.

Subjects fulfilling inclusion criteria were eligible for NTS. Catheters were inserted in a random order to minimize selection bias and carryover effect: 20 sealed envelopes ( 1 for each subject) were prepared by a team not related to the study. These envelopes contained a paper with either the letter $\mathrm{T}$, representing a curved edge catheter or $\mathrm{N}$, representing a conventional suction catheter, indicating the type of catheter that would first be applied.

\section{Technique}

Each subject was comfortably placed in bed and, if possible, she/he was offered reassurance concerning the procedure. Whenever appropriate, a pillow was placed beneath the occiput, displacing the cervical spine anteriorly, to align 3 axes: those of the mouth, oropharynx, and trachea. Suctioning equipment was prepared as outlined by Moore ${ }^{15}$ and presented in American Association for Respiratory Care guidelines. ${ }^{16}$ The nostril contralateral to the one with the nasogastric tube was selected. The oxygen mask was maintained throughout the procedure to avoid hypoxemia. In all cases, the same person was performing the nasotracheal insertion.

A different person/observer (but the same in all cases) recorded the measurements, counting the attempts as well as determining the exact placement of the catheter into the trachea using a stethoscope. This person did not know the type of catheter being used each time. He participated in the procedure after the end point of the catheter was inserted into the nostril, so it was impossible to distinguish the type of catheter used because the 2 catheters are identical except for the end point. The suctioning wall device had, as usual, a negative pressure of $200 \mathrm{~mm} \mathrm{Hg}$ as provided by the hospital protocol for all endotracheal suctions.

\section{Passing Nasopharynx}

After lubricating the catheter's tip with $2 \%$ lidocaine lubricant, the catheter was directed in a ventral and slightly medial orientation toward the ventral nasal meatus. The passage of the nasopharynx was determined by the depth of the catheter in the nostril. After $5 \mathrm{~cm}$, the passage was assumed to be successful. In all cases, the difficulty of the passage through the turbinates was at $2-3 \mathrm{~cm}$.

If the catheter met firm resistance, indicative of its placement in the dorsal meatus, it was pulled slightly outward and was redirected inward. The time required to pass through the nasopharynx was recorded as T1. No more than 5 attempts were made, as per the protocol design.

\section{Entering Trachea}

To help the catheter insertion, subjects were asked, when it was feasible, to inhale while the catheter was pushed gently into the trachea. While using the curved edge catheter, the curved tip of the catheter was carefully turned anteriorly to check whether this would provide additional help in easing the insertion into the trachea.

Successful insertion was confirmed if: (1) another member of the team could hear turbulent flow on lung auscultation while applying suction; (2) there was coughing upon insertion; (3) there was no resistance during insertion. On the contrary, insertion was recorded as unsuccessful if 
there was no turbulence on auscultation, and subjects were grimacing instead of coughing. In the second case, the catheter was pulled slightly backward (approximately $1 \mathrm{~cm}$ ) and redirected forward to the trachea. The time required to pass from the nasopharynx to the trachea was recorded as T2. No more than 10 attempts were made (a cutoff selected mostly by experience) as per the protocol design. Each micromanipulation was counted as 1 individual attempt (and not the reinsertion of the catheter from the beginning of the procedure). The success entering into the trachea, the number of attempts, and the process time were recorded by a member of the team who was blinded to the type of catheter used each time.

It should be noted that (1) the same particular professional was performing all attempts of the nasotracheal insertion in all cases of the study; (2) the professional who performed the procedure was skilled in nasotracheal suction and had no experience (except for a pilot study) in suctioning with Tiemann type catheters; (3) the professional who performed the procedure was a member of the research group, and all of the members of the research group are members of clinical staff as well.

To avoid an expectancy bias, a different person/observer (but the same in all cases) recorded the measurements, counted the attempts, and determined the exact placement of the catheter into the trachea using a stethoscope. This person did not know the type of catheter used each time. He participated in the procedure after the end point of catheter insertion into the nostril, and it was impossible to distinguish the kind of catheter because the 2 catheters are identical except for the end point. Thus, the number of attempts and the definition of entry in the nasopharynx were determined with a blind procedure.

\section{Other Parameters Recorded}

On the recruitment day, the sex, age, number of days in ICU, primary reason for ICU admission, Sequential Organ Failure Assessment score, and type of catheter first used were recorded for each subject. Moreover, the following parameters were recorded, before and after each NTS: $\mathrm{F}_{\mathrm{IO}_{2}}, \mathrm{~S}_{\mathrm{pO}_{2}}$ (by pulse oximetry), breathing frequency, heart rate, and systolic/diastolic blood pressure. Finally, the presence of blood on the catheter tip was recorded with a simple yes or no, at the end of the procedure.

\section{Statistical Methods}

Sample Size. In the crossover study, the 2 interventions were applied sequentially to the same subject, on the same day and after a minimum time interval of 30 min. ${ }^{12,13} \mathrm{~A}$ pilot study was first conducted with 10 subjects. Data from this pilot study were not included in the main study. The pilot study was a training period for the procedure, and throughout this period were recorded all data considered useful only for determining the sample size of the main study. Those data were not sufficient to be used in the main study. The amount of acceptable mean difference between the curved edge catheter and conventional suction catheter technique is the number of attempts or mean differences of required time for tracheal access.

Calculating Sample Size From Mean Difference of Number of Attempts. For population SD $(\sigma 1)$ of 3.9, type I $(\alpha)$ error of 0.05 power of 0.8 , and mean difference 4.3 , a sample size of 20 subjects was sufficient. ${ }^{17}$

Calculating Sample Size From Mean Difference of Required Time. Considering the sample size calculation from mean differences of required time $(8.8 \mathrm{~s}$ for the curved edge catheter and $21.3 \mathrm{~s}$ for the conventional suction catheter) for $\mathrm{SD}(\sigma 2)$ of 11.3 , type I $(\alpha)$ error of 0.05 , and power of 0.8 , it was determined that a sample size of 20 subjects was sufficient as well. ${ }^{17}$

Data Analysis. Data were analyzed using standard statistical methods and 95\% CIs. The Kolmogorov-Smirnov test was applied to test data for normality. If the groups of data were normally distributed, then the Student paired $t$ test was adopted to evaluate the difference between the means for $\mathrm{S}_{\mathrm{pO}_{2}}$, heart rate, breathing frequency, systolic pressure, and diastolic pressure. The above values were interdependent and related to paired measurements (before and after intervention) on the same person. If not, a nonparametric Wilcoxon rank-sum test was used. Therefore, results were presented as mean values \pm SD and median values (range), respectively. The effect size was calculated using an odds ratio. For paired nominal parameters, the McNemar chi-square test was adopted. To determine the difference in process time between the 2 catheters, Kaplan-Meier analysis and long-rank test were used. In this study, Kaplan-Meier analysis was considered appropriate because it gives quick and comparative information about the time spent applying the 2 methods of tracheal access as well as about the effectiveness of tracheal access.

For statistical analysis, SPSS 20 (SPSS, Chicago, Illinois) was used. All statistical tests were 2 -sided and conducted at a $P<.05$ level of significance.

\section{Results}

The study was conducted from May through September 2014. During this time period, the Physiotherapy Department provided 416 chest physiotherapy sessions in 83 non-intubated ICU patients. Forty-three patients were excluded because their bronchial secretions were managed noninvasively, 5 patients were excluded due to bleeding disorders, and 5 patients were excluded due to rapid clin- 


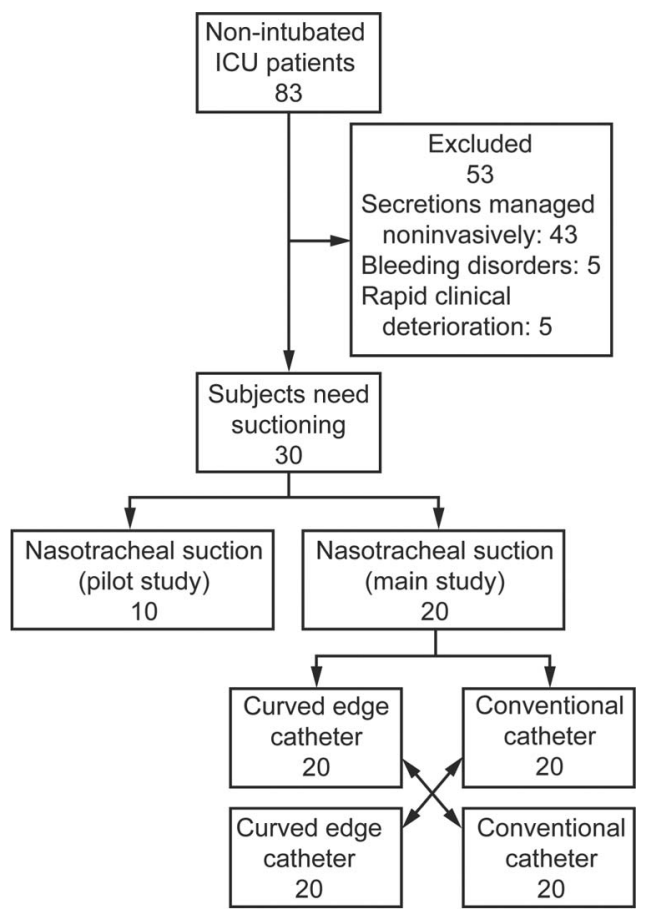

Fig. 2. Flow chart.

ical deterioration that demanded endotracheal intubation. Thus, 60 NTSs were performed in a total of 30 subjects, 20 for the pilot study and 40 for the main study, in 10 and 20 subjects, respectively. The main study included 20 subjects (10 men and 10 women), with a mean age of $75.5 \pm 10.1$ y (Fig. 2, Table 1).

To determine the success rate of each catheter, the number of tracheal insertions was calculated over the total number of attempts. In one subject, it was not possible to pass through the nostril using either catheter. The overall success rate for the curved edge catheter was 19/52 (successful/unsuccessful attempts) $(36.5 \%, 95 \%$ CI $23.6-$ $51.0 \%$ ), whereas the success rate for the conventional suction catheter was $12 / 130(9.2 \%, 95 \%$ CI $4.8-15.5 \%)$. The curved edge catheter was 5.7 times more likely to achieve tracheal insertion compared with the conventional suction catheter (odds ratio $5.66,95 \%$ CI $2.49-12.84, P<.001$ ).

The traumatic rate is the percentage of catheters with blood present (on the tip of each catheter/blood streaks) in the total population. This percentage was equal for both catheters, 30\% (6/20) (Tables 2, 3, and 4).

Figures 3 and 4 show a graphic illustration of the attempts and the time needed to pass through the nasopharynx and to proceed from the nasopharynx to the trachea with the curved edge catheter and the conventional suction catheter, respectively.

Finally, in Figure 5, Kaplan-Meier analysis shows time differences in achieving passage through the nasopharynx (Fig. 5A) and insertion into the trachea (Fig. 5B), referring
Table 1. Subjects' Characteristics

\begin{tabular}{lc}
\hline \multicolumn{1}{c}{ Characteristic } & Values \\
\hline Age, mean \pm SD y & $75.5 \pm 10.1$ \\
Male subjects, $n$ & 10 \\
SOFA score on intervention day, mean \pm SD & $4.4 \pm 2.1$ \\
No. of ICU intervention days, mean \pm SD & $19.2 \pm 17.9$ \\
Primary reason for ICU admission, $n(\%)$ & \\
Coronary artery bypass graft & $3(15)$ \\
Aortofemoral bypass surgery & $1(5)$ \\
Cardiac valve replacement & $2(10)$ \\
Hemopericardium & $1(5)$ \\
Abdominal cancer & $3(15)$ \\
Heart failure & $4(20)$ \\
Traumatic brain injury & $2(10)$ \\
Meningitis & $1(5)$ \\
COPD, ARF & $3(15)$ \\
& \\
$N=20$. & \\
SOFA Sequential Organ Failure Assessment & \\
ARF $=$ acute respiratory failure & \\
\hline
\end{tabular}

to each catheter. The log-rank test, for both passing the nasopharynx $\left(P_{1}\right)$ and reaching the trachea $\left(P_{2}\right)$ showed that the curved edge catheter requires a significantly shorter time $\left(P_{1}=0.04\right.$ and $P_{2}=0.005$, respectively).

\section{Additional Calculations}

To determine whether there was any difference between the first and later attempts, the correlation between subject regression and the number of attempts with both catheters was investigated and showed that there was moderate ( -0.57 , curved edge catheter) to weak correlation $(-0.26$, conventional suction catheter). (1) The success rate of the first attempt was $7 / 52=0.13$ or $13 \%$ when using the curved edge catheter, whereas it was $3 / 130=0.02$ or $2 \%$ when using the conventional suction catheter. (2) The success rate without the first attempt was $11 / 33=0.33$ or $33 \%$ when using the curved edge catheter, whereas it was $8 / 46=0.17$ or $17 \%$ when using the conventional suction catheter. (3) The catheter was placed correctly at a rate of $18 / 20=0.9$ or $90 \%$ when using the curved edge catheter, whereas the rate was $11 / 20=0.55$ or $55 \%$ when using the conventional suction catheter.

\section{Discussion}

The curved edge catheter seemed to be more effective than the conventional suction catheter on 2 main points: success rate and time needed to insert to the pharynx and trachea. Anatomically, the most difficult point to access between the trachea and pharynx is the trachea. 
Table 2. Number of Attempts Needed to Access Nasopharynx and Trachea by Inserting Curved Edge Catheters and Conventional Suction Catheters

\begin{tabular}{|c|c|c|c|c|c|c|}
\hline & NP by CEC & $\mathrm{NP}$ by CSC & $P$ & Trachea by CEC & Trachea by CSC & $P$ \\
\hline No. of attempts, median (range) & $1(1)$ & $2.5(8)$ & .001 & $2(9)$ & $9(9)$ & .002 \\
\hline $\begin{array}{l}\mathrm{NP}=\text { nasopharyn } \\
\mathrm{CEC}=\text { curved edge catheter } \\
\mathrm{CSC}=\text { conventional suction } \text { catheter }\end{array}$ & & & & & & \\
\hline
\end{tabular}

Table 3. Time Needed to Access Nasopharynx and Trachea by Inserting Curved Edge Catheters and Conventional Suction Catheters

\begin{tabular}{|c|c|c|c|c|c|c|}
\hline & NP by CEC & NP by CSC & $P$ & Trachea by CEC & Trachea by CSC & $P$ \\
\hline Time, median (range) s & $3(11)$ & $5.3(18)$ & .038 & $6(27)$ & $20(25)$ & .002 \\
\hline $\begin{array}{l}\mathrm{NP}=\text { nasopharynx } \\
\mathrm{CEC}=\text { curved edge catheter } \\
\mathrm{CSC}=\text { conventional suction cathe }\end{array}$ & & & & & & \\
\hline
\end{tabular}

Table 4. Secondary Parameters and Physiological Impairment Related to Nasotracheal Suctioning by Curved Edge and Conventional Suction Catheters

\begin{tabular}{lcr}
\hline \hline \multicolumn{1}{c}{ Parameter } & Curved Edge Catheter & Conventional Suction Catheter \\
\hline $\mathrm{S}_{\mathrm{pO}_{2}, \text { mean } \pm \mathrm{SD} \%}$ & & $P$ \\
$\quad$ Before procedure & $96.6 \pm 2.9$ & $96.3 \pm 3$ \\
$\quad$ After procedure & $96.2 \pm 3$ & $95.7 \pm 3.8$ \\
Breathing frequency, mean \pm SD breaths/min & & $22 \pm 4.9$ \\
$\quad$ Before procedure & $22.7 \pm 7.9$ & $25.8 \pm 7.7$ \\
$\quad$ After procedure & $26.1 \pm 7.4$ & $82.4 \pm 13.2$ \\
Heart rate, mean \pm SD beats/min & & $87.7 \pm 14.2$ \\
$\quad$ Before procedure & $81.7 \pm 13$ & $134.7 \pm 22.5$ \\
$\quad$ After procedure & $88.2 \pm 15.4$ & $141.5 \pm 18.9$ \\
SBP, mean \pm SD mm Hg & & .55 \\
$\quad$ Before procedure & $133.2 \pm 22.8$ & .79 \\
$\quad$ After procedure & $142.7 \pm 23.6$ & .68 \\
Traumatic rate, $\%$ & 30 (Tiemann) & .66 \\
$\quad$ Evidence of blood on catheter's tip & & .45 \\
SBP $=$ systolic blood pressure & & $>.99$ \\
\hline
\end{tabular}

Gaining access to the trachea is not an easy procedure and requires several attempts. Nevertheless, it was found that the curved edge catheter has an overall success rate of $36.5 \%$, comparing favorably with the only $9.2 \%$ success rate of the conventional suction catheter. Indeed, the curved edge catheter was found to be approximately 6 times more likely to insert into the trachea compared with the conventional suction catheter. Past literature provides very little relevant information. In fact, there is only 1 published report in which the authors stated (without statistical evidence) that the curved edge catheter was superior to the conventional suction catheter in achieving tracheal catheterization, in 120 subjects with early postoperative respiratory failure. ${ }^{9}$ The Tiemann type catheter was designed for urethral intubation. This fact ex- plains the reason why there was no further information in the literature about the use of this type of catheter in suctioning.

Moreover, although one might assume the angular distal edge of the curved edge catheter to be more traumatic, this was not demonstrated by the results of the present study. Finally, one could argue in favor of the curved edge catheter because the smaller number of attempts required reduces the risk for mucosal injury.

In the present crossover study, each subject served as his own control; therefore, inter-individual variability was avoided. Confounding factors that would affect the NTS technique were also eliminated, since the interventions were applied sequentially in all subjects. Catheters were also inserted in a random order so as to minimize 


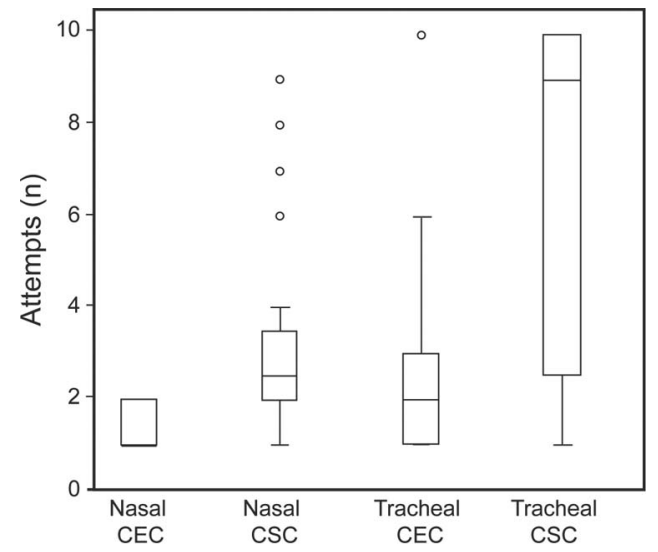

Fig. 3. Box and whisker plot as a schematic representation of the attempts needed to pass through the nasopharynx and to proceed from the nasopharynx to the trachea with curved edge catheters (CEC) and conventional suction catheters (CSC). Boxes represent interquartile ranges; the center line across each box is the median. Whiskers extend to the highest and lowest values, with outliers shown as circles.

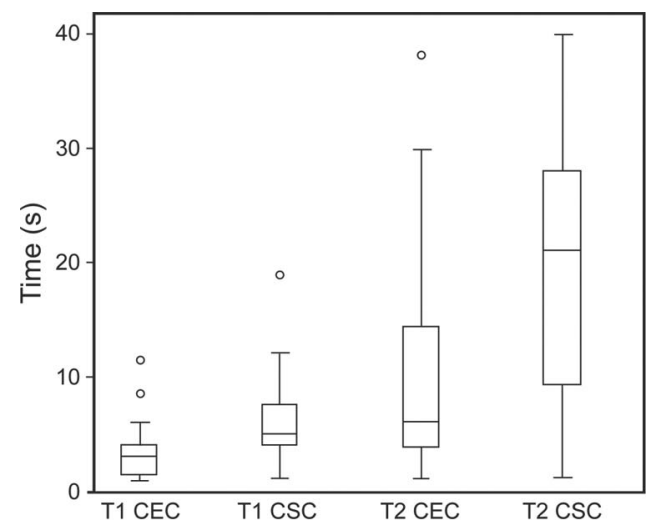

Fig. 4. Box and whisker plot as schematic representation of the time needed to pass through the nasopharynx (T1) and to proceed from the nasopharynx to the trachea (T2) with curved edge catheters (CEC) and conventional suction catheters (CSC). Boxes represent interquartile ranges; the center line across each box is the median. The whiskers extend to the highest and lowest values, with outliers shown as circles.

selection bias and carryover effect, often encountered in crossover studies.

Because of the above considerations, it is assumed that the findings of the current study could be of clinical importance for the management of the non-intubated critically ill patient with respiratory failure, in whom therapeutic interventions should be practical, rapid, effective, and cost-effective (the cost of the curved edge catheter in Europe is $€ 0.01$ greater than that of the conventional suction catheter).

Indeed, in ICU settings, time is an important issue: The vast majority of NTS is emergently indicated, and time pressure, situational stress, and the patient's ongoing clin-
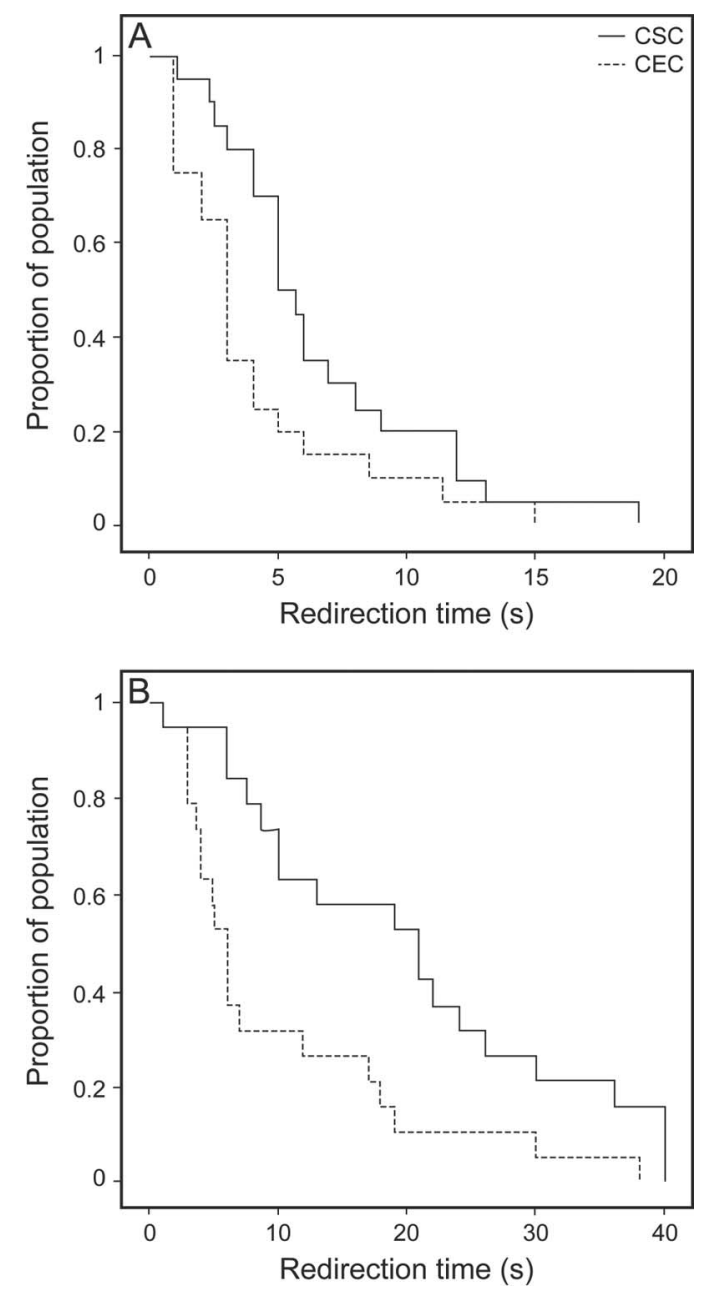

Fig. 5. Kaplan-Meier analysis shows differences in achieving passage through the nasopharynx by a curved edge catheter (CEC) and a conventional suction catheter (CSC) (A). Kaplan-Meier analysis shows differences for insertion into trachea by the curved edge catheter and the conventional suction catheter (B).

ical deterioration dictate the application of an intervention of maximal effect.

Correlation between subject regression and the number of attempts with both catheters showed that there was moderate $(-0.57$, curved edge catheter) to weak correlation $(-0.26$, conventional suction catheter). This correlation means that expertise improved with greater use of the curved edge catheter, compared to the conventional suction catheter, where expertise remained unchanged.

Additional calculations showed that: (1) one-third of first attempts succeeded at insertion into the trachea; (2) the curved edge catheter was placed correctly in almost all cases $(90 \%)$, whereas the conventional suction catheter placement failed in $45 \%$ of cases. Maximum and minimum values of parameters in Table 4 help to clarify the safety data. 
In the case of $\mathrm{S}_{\mathrm{pO}_{2}}$, there was a minimum saturation value of $90 \%$ for the curved edge catheter, whereas the value for the conventional suction catheter was $87 \%$. This evidence showed that there were no severe desaturations. There were no extreme cases of systolic blood pressure $(>180 \mathrm{~mm} \mathrm{Hg}$ ) except for 1 case in which the pressure was $184 \mathrm{~mm} \mathrm{Hg}$. There were no cases of severe arrhythmia, such as atrial fibrillation or ventricular extrasystoles. Subject discomfort was not evaluated due to communication problems. A significant percentage of the pilot study subjects (4/10) were not fully conscious, and the Glasgow coma score for these subjects was found to be $\leq 14$.

\section{Limitations}

The main limitation for this study was the absence of literature regarding usage of the curved edge catheter in suctioning. This was to be expected because the main usage of this type of catheter is in urology and not in respiratory care. The fact that the professional who was performing suctioning knew the type of catheter used each time was of course another limitation of the study. To overcome this, a different person/observer (but the same in all cases) who was recording the measurements, counting the attempts, and determining the exact placement of the catheter into the trachea did not know the type of catheter being used each time. He participated in the procedure after the end point of catheter insertion into the nostril, and it was impossible to distinguish the kind of catheter because the 2 catheters are identical except for the tip.

Finally, the pilot study results showed that ICU subjects who needed NTS were confused at a rate of $4 / 10$. The Glasgow coma score for those 4 subjects was $\leq 14$. This rate makes questionable the ability to give conscious answers, and it was considered not statistically usable.

\section{Conclusions}

In NTS, a curved edge catheter is approximately 6 times more likely to enter the trachea compared with a conventional suction catheter. In NTS, the curved edge catheter requires fewer attempts and a shorter process time to reach the trachea in comparison with the conventional suction catheter, and this probably reduces the potential risk of nasal and tracheal injury. In NTS, the curved edge catheter seems to be as safe as the conventional suction catheter.

In conclusion, the curved edge catheter could be used alternatively to the conventional suction catheter for NTS, because its application in critically ill subjects was found to be more successful and rapid. It is recommended for cases where a standard catheter fails to access the trachea or in cases where the patient's clinical condition demands a fast and accurate intervention.

\section{REFERENCES}

1. Toomtong P, Raksakietisak M, Vorakitpokatorn P. Clinical outcomes of failed extubation in a postoperative intensive care unit. $\mathrm{J}$ Med Assoc Thai 2002;85(Suppl 3):S987-S992.

2. Konrad F, Schreiber T, Brecht-Kraus D, Georgieff M. Mucociliary transport in ICU patients. Chest 1994;105(1):237-241.

3. Hess D, MacIntyre NR, Mishoe SC, Galvin WF, Adams AB. Respiratory care: principles and practice. 2nd edition. Burlington, Massachusetts: Jones \& Bartlett Learning; 2011:366.

4. Gosselink R, Bott J, Johnson M, Dean E, Nava S, Norrenberg M, et al. Physiotherapy for adult patients with critical illness: recommendations of the European Respiratory Society and European Society of Intensive Care Medicine Task Force on physiotherapy for critically ill patients. Intensive Care Med 2008;34(7):1188-1199.

5. Orebaugh SL, Bigeleisen PE. Atlas of airway management: techniques and tools. 1st edition. Philadelphia: Lippincott Williams \& Wilkins; 2007:81-84.

6. Strickland SL, Rubin BK, Drescher GS, Haas CF, O'Malley CA, Volsko TA, et al. AARC clinical practice guideline: effectiveness of nonpharmacologic airway clearance therapies in hospitalized patients. Respir Care 2013;58(12):2187-2193.

7. Meyer P, Rousseau H, Maillet JM, Thierry S, Sy O, Vicaut E, et al. Evaluation of blind nasotracheal suctioning and non-bronchoscopic mini-bronchoalveolar lavage in critically ill patients with infectious pneumonia: a preliminary study. Respir Care 2014;59(3):345-352.

8. Kazmierska K, Szwast M, Ciach T. Determination of urethral catheter surface lubricity. J Mater Sci Mater Med 2008;19(6):2301-2306.

9. Pode D, Manny J. A simplified method for repeated nasotracheal suction. Surg Gynecol Obstet 1984;159(2):173-174.

10. Wang B-S, Wang X-J, Gong L-K. The construction of a Williams design and randomization in cross-over clinical trials using SAS. J Stat Softw 2009;29:1-10.

11. World Medical Association. Declaration of Helsinki: ethical principles for medical research involving human subjects. Bull World Health Organ 2001;79(4):373-374.

12. Sook MS, Sook PK. A comparison of the opened versus closedsystem of suctioning in oxygen saturation, vital signs and suction time. Korean J Adult Nurs 2009;21(2):141-154.

13. Afshari A, Safari M, Oshvandi K, Soltanian AR. The effect of the open and closed system suctions on cardiopulmonary parameters: time and costs in patients under mechanical ventilation. Nurs Midwifery Stud 2014;3(2):e14097.

14. Gupta A, Epstein JB, Cabay RJ. Bleeding disorders of importance in dental care and related patient management. J Can Dent Assoc 2007; 73(1):77-83.

15. Moore T. Suctioning techniques for the removal of respiratory secretions. Nurs Stand 2003;18(9):47-53.

16. American Association for Respiratory Care. Clinical practice guideline: nasotracheal suctioning-2004 revision \& update. Respir Care 2004;49(9):1080-1084.

17. Julious SA. Sample sizes for clinical trials with normal data. Stat Med 2004;23(12):1921-1986. 